\title{
Proteomics of anatomical sections of the gut of Nosema-infected Western honey bee (Apis mellifera) reveals different early responses to Nosema spp. isolates
}

${ }^{1,2}$ Camille Houdelet, ${ }^{3}$ Chainarong Sinpoo, ${ }^{3}$ Thunyarat Chantaphanwattana, ${ }^{2}$ Sébastien N. Voisin, ${ }^{4}$ Michel Bocquet, ${ }^{3,5}$ Panuwan Chantawannakul* and ${ }^{1,2}$ Philippe Bulet*.

${ }^{1}$ CR University Grenoble Alpes, Institute for Advanced Biosciences, Inserm U1209, CNRS UMR 5309, Grenoble, France

${ }^{2}$ Plateform BioPark Archamps, 260 Avenue Marie Curie Archparc 74166 Saint Julien-en Genevois France

${ }^{3}$ Department of Biology, Faculty of Science, Chiang Mai University, Chiang Mai 50200, Thailand

${ }^{4}$ Michel Bocquet, Apimedia, Annecy, France

${ }^{5}$ Environmental Science Research Center (ESRC), Faculty of Science, Chiang Mai

University, Thailand

\section{Table of content}

Figure S1. Experimental procedure to infect individual bees with a Nosema inoculum......S-2

Figure S2. KEGG pathways of the up-regulated proteins

Figure S3. KEGG pathways of the down-regulated proteins. S-6-8

Table S1. PCR results of the identification of the four Nosema isolates.

Table S2. Nosema spores counted for each experimental modality S-10

Table S3. Multireport of proteins identified in the esophagus section. (xlsx)

Table S4. Multireport of proteins identified in the crop section of the Apis mellifera gut tissue. $(x 1 s x)$

Table S5. Multireport of proteins identified in the midgut section. (xlsx)

Table S6. Multireport of proteins identified in the ileum section. (xlsx)

Table S7. Multireport of proteins identified in the rectum section. (xlsx)

Table S8. Comparison of the proteins identified between our experiments and previous published data. (xlsx) 


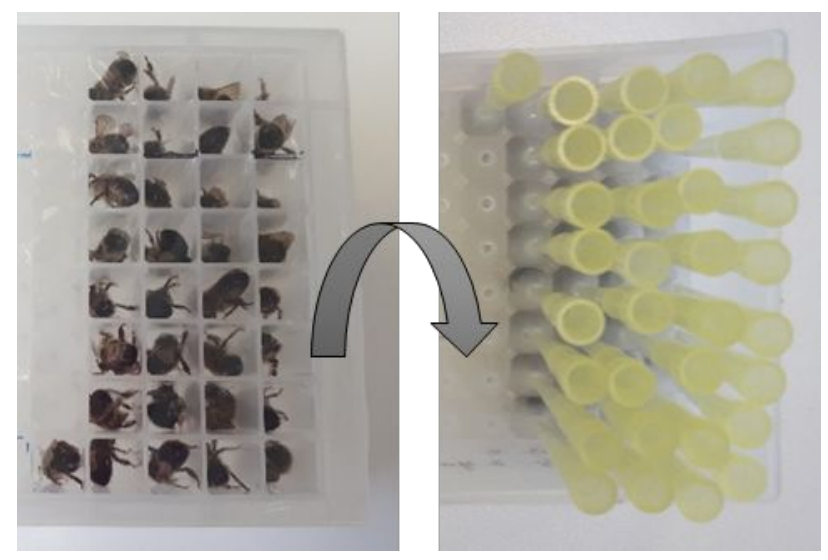

Figure S1: Experimental procedure to infect individual bees with a Nosema inoculum To infect a large number of bees individually with a Nosema inoculum, we have developed the following method. After 15 hours of fasting each bee was place individually in a homemade "inoculated-96-well deep bottom plate". It consists of a 96-well deep bottom plate with holes at the bottom. After placing the bees in the holes, the box is covered with a plastic film. The box is turned over, then 10-200 $\mu \mathrm{L}$ pipette tips containing the syrup are placed in front of each bee. Bees were either fed with $10 \mu \mathrm{L}$ of $50 \%$ syrup containing 100,000 spores for the experimental bees, or fed with syrup alone for the control bees. The bees were kept in this device the time to consume all of the syrup. They were then removed from the device and then placed by experimental group in boxes with syrup ad libitum until tissues collection. 
Figure S2: Mapping of the up-regulated proteins of $A$. mellifera in a context of infection by Nosema spp. isolates.

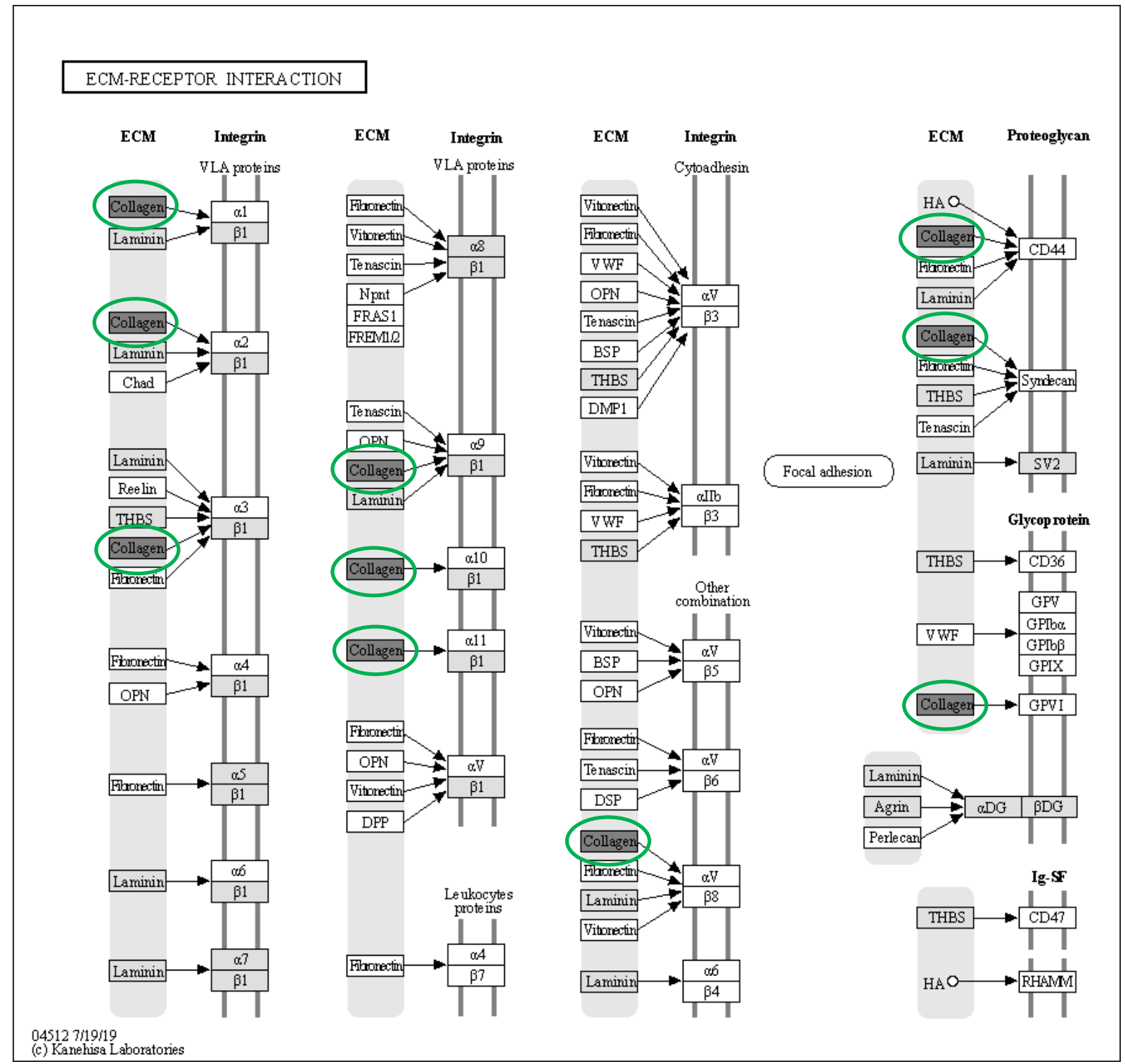

S2A: Mapping of the pathway ECM-receptor interaction (KEGG Pathways number ame04512). In a presence of Nosema spores the two Collagen IV NC1 domain-containing proteins of $A$. mellifera upregulated are involved in the protein processing in endoplasmic reticulum pathway (in green circles). 


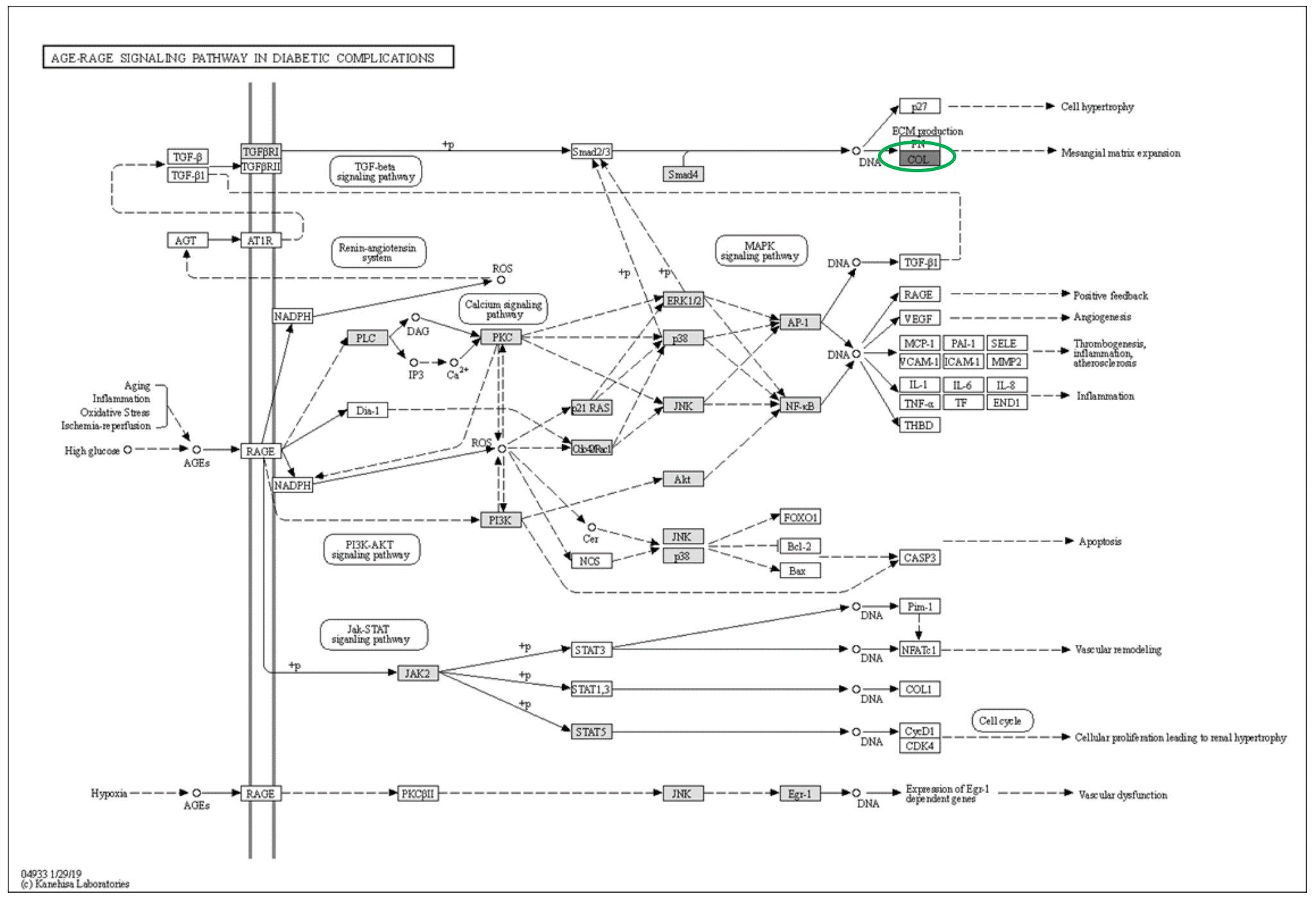

S2B: Mapping of the pathway AGE-RAGE signaling pathway in diabetic complications (KEGG Pathways number ame04933). In a presence of Nosema spores the two Collagen IV NC1 domain-containing proteins of $A$. mellifera up-regulated are involved in the protein processing in endoplasmic reticulum pathway (in green circle). 


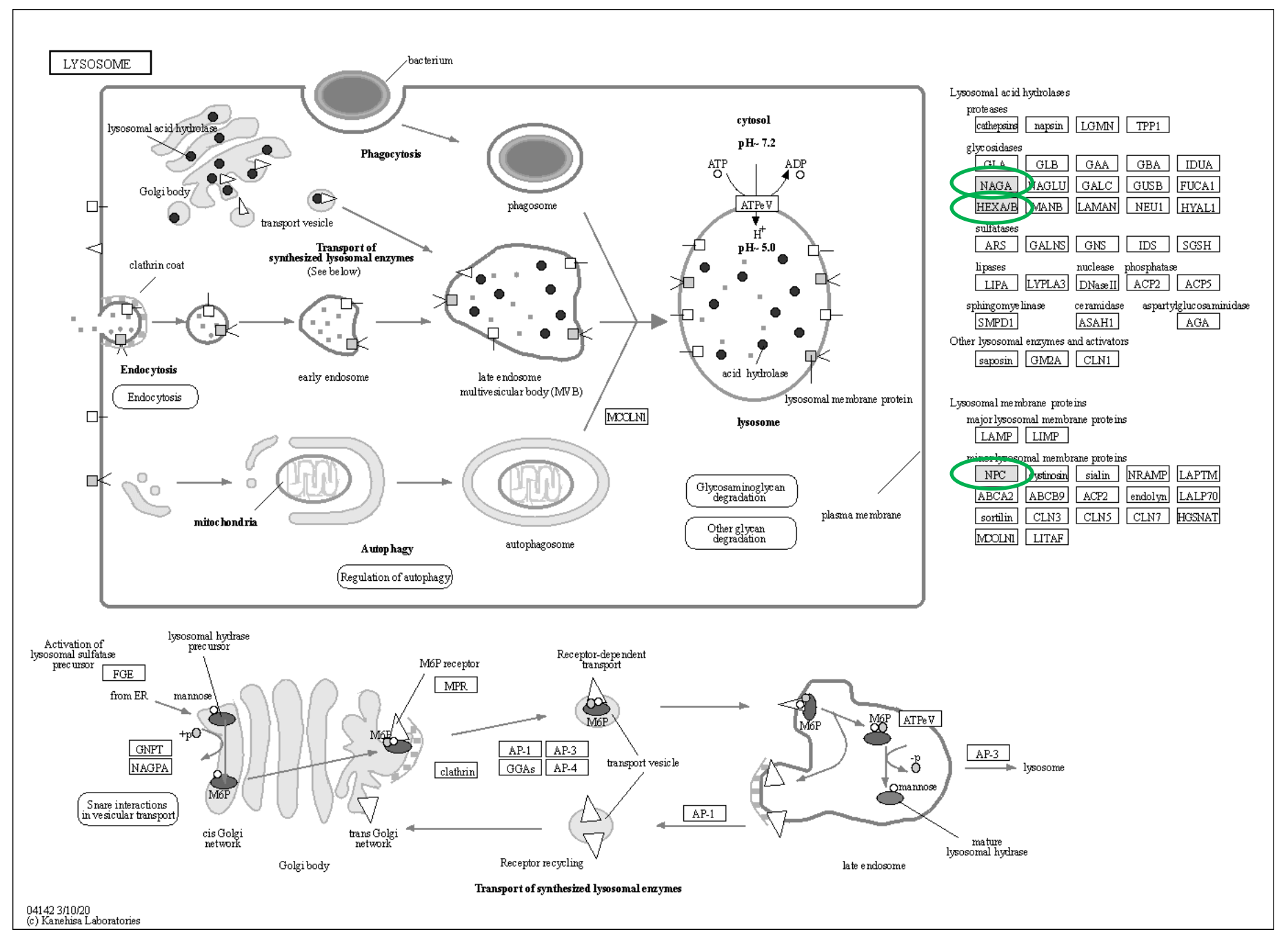

S2C: Mapping of the lysosome pathway (KEGG Pathways number ame04933). In a presence of Nosema spores the three proteins (Alpha-galactosidase, Betahexosaminidase and $\mathrm{ML}$ domain-containing protein) of $A$. mellifera up-regulated are involved in the protein processing in endoplasmic reticulum pathway 
Figures S3: Mapping of the down-regulated proteins of $\boldsymbol{A}$. mellifera in a context of infection by Nosema spp. isolates.

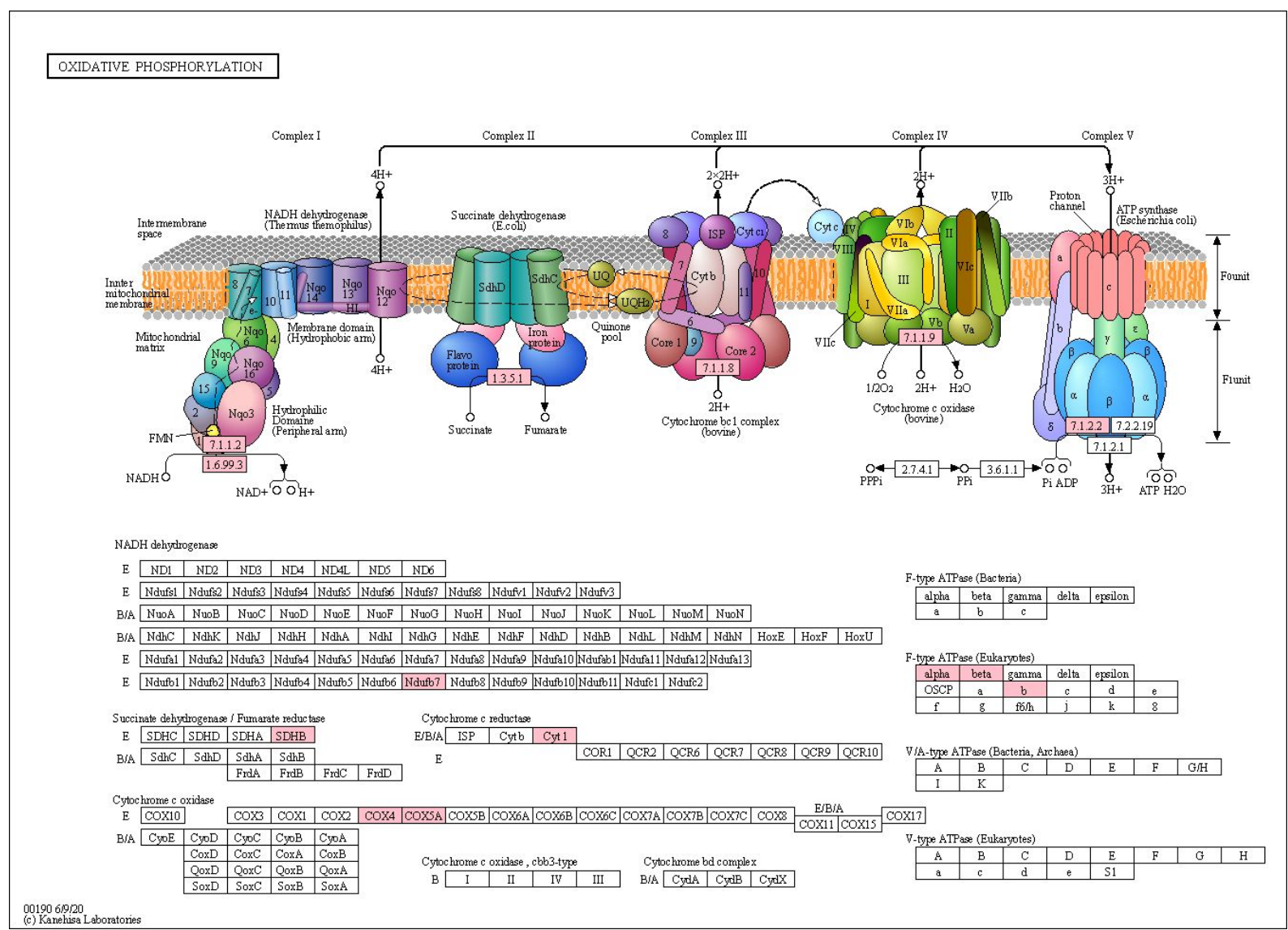

S3A: Mapping of the pathway oxidative phosphorylation (KEGG Pathways number ame00190). In a presence of Nosema spores the eight proteins (ATP synthase subunit b, cytochrome $c$ oxidase subunit 5A, ATP synthase subunit alpha, cytochrome $c$ oxidase subunit 4 isoform 1 , cytochrome $c 1$, succinate dehydrogenase iron-sulfur subunit, ATP synthase subunit beta, NADH dehydrogenase 1 beta subcomplex subunit 7) of $A$. mellifera down-regulated are involved in the oxidative phosphorylation pathway (in red). 


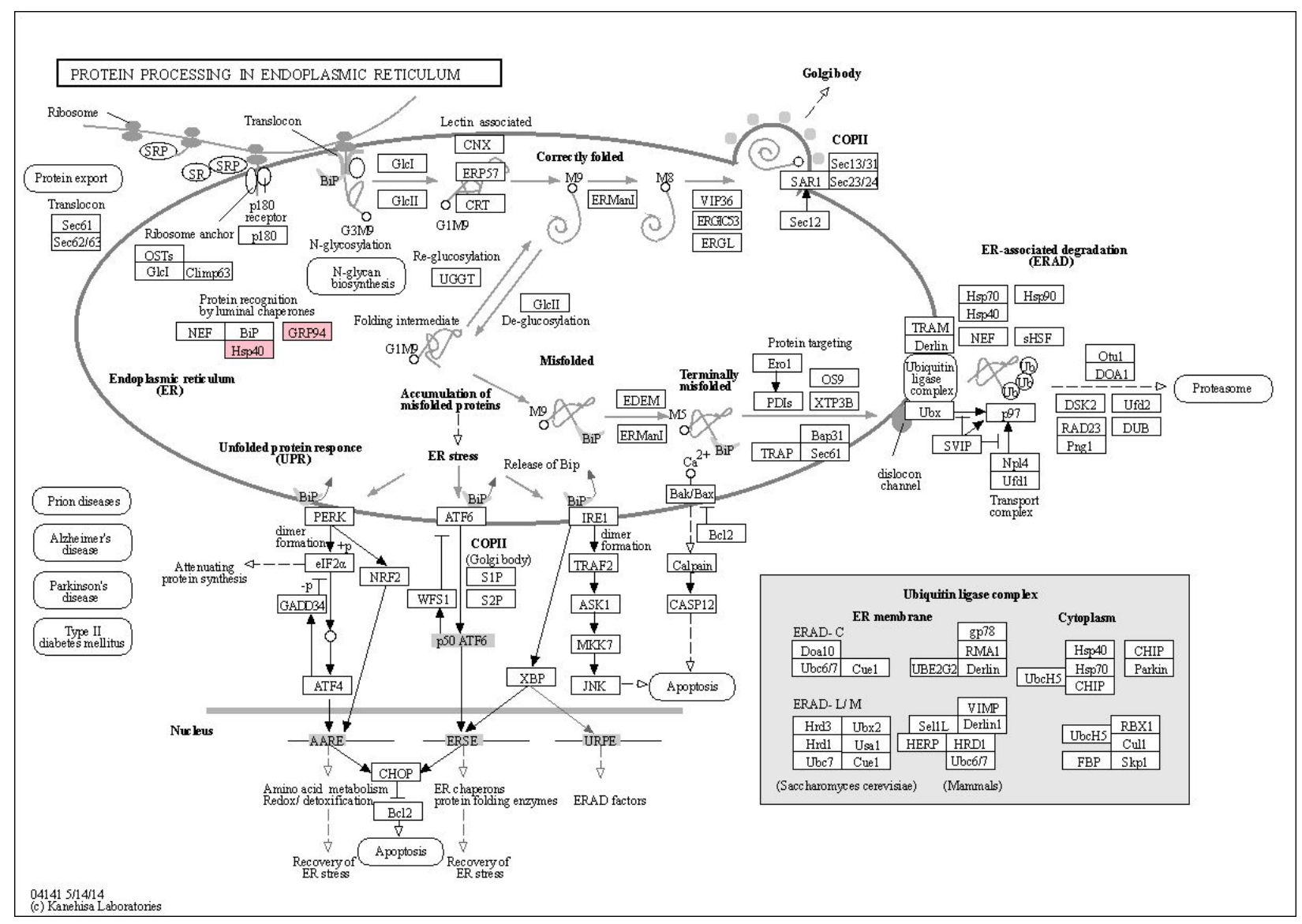

S3B: Mapping of the pathway protein processing in endoplasmic reticulum (KEGG Pathways number ame04141). In a presence of Nosema spores the two proteins (HATPase_c domain-containing protein and Uncharacterized protein with the accession number A0A088ABL3) of A. mellifera down-regulated are involved in the protein processing in endoplasmic reticulum pathway (in red). 


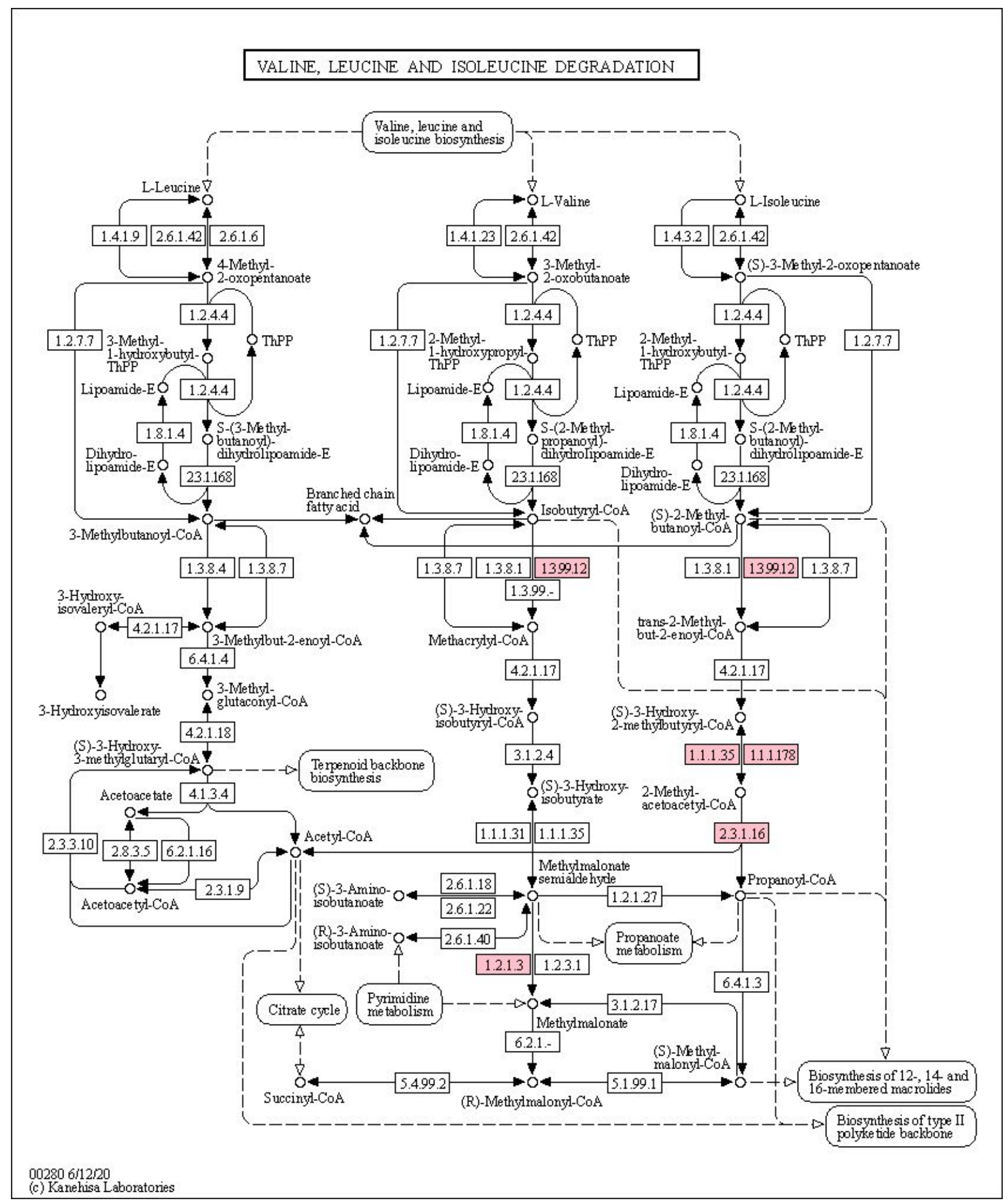

S3C: Mapping of the pathway valine, leucine and isoleucine degradation (KEGG Pathways number ame00280). In a presence of Nosema spores the four proteins (short/branched chain specific acyl-CoA dehydrogenase, aldehyde dehydrogenase, trifunctional enzyme subunit beta,3-hydroxyacyl-CoA dehydrogenase type-2) of $A$. mellifera down-regulated are involved in the oxidative phosphorylation pathway

red). 
Table S1: PCR results of the four Nosema isolates.

Nosema species were confirmed by PCR analysis. The presence of Nosema was scored as 1 and 0 for its absence.

\begin{tabular}{|c|c|c|c|}
\hline \multicolumn{4}{|c|}{ Apis mellifera } \\
\hline \multirow[t]{3}{*}{ Condition of Nosema infection } & \multirow{3}{*}{ Apiary } & \multicolumn{2}{|c|}{ PCR confirmation } \\
\hline & & & \\
\hline & & N. ceranae & N. apis \\
\hline \multirow{3}{*}{$\begin{array}{l}\text { N. ceranae from } A \text {. cerana from Thailand } \\
\text { (NC1) }\end{array}$} & 1 & 1 & 0 \\
\hline & 2 & 1 & 0 \\
\hline & 3 & 1 & 0 \\
\hline \multirow{3}{*}{$\begin{array}{l}\text { N. ceranae from } A \text {. mellifera from Thailand } \\
\text { (NC2) }\end{array}$} & 1 & 1 & 0 \\
\hline & 2 & 1 & 0 \\
\hline & 3 & 1 & 0 \\
\hline \multirow[t]{3}{*}{ N. ceranae from A. mellifera from France (NF) } & 1 & 1 & 0 \\
\hline & 2 & 1 & 0 \\
\hline & 3 & 1 & 0 \\
\hline \multirow[t]{3}{*}{ N. apis (NA) } & 1 & 0 & 1 \\
\hline & 2 & 0 & 1 \\
\hline & 3 & 0 & 1 \\
\hline
\end{tabular}


Table S2: Nosema spores counted for each experimental modality.

\begin{tabular}{|c|c|c|c|c|c|}
\hline \multicolumn{6}{|c|}{ Apis mellifera } \\
\hline \multirow[t]{2}{*}{ Nosema spp. } & \multirow[t]{2}{*}{ Apiary } & & & & \\
\hline & & Result & MEAN & SD & SE \\
\hline \multirow{8}{*}{$\begin{array}{l}\text { N.ceranae from } \\
\text { A. cerana from } \\
\text { Thailand } \\
\text { (NC1) }\end{array}$} & & $27,600,000$ & $25,366,666.67$ & $4,488,132.50$ & $2,591,224.50$ \\
\hline & 1 & $28,300,000$ & & & \\
\hline & & $22,900.000$ & $24,433.333 .33$ & 1418.919 .77 & 81921371 \\
\hline & 2 & $25,700,000$ & & & \\
\hline & & $24,700,000$ & & & \\
\hline & & $22,700,000$ & $23,266,666.67$ & $1,530,795.00$ & $883,804.90$ \\
\hline & 3 & $22,100,000$ & & & \\
\hline & & $25,000,000$ & & & \\
\hline \multirow{8}{*}{$\begin{array}{l}\text { N.ceranae from } \\
\text { A. mellifera } \\
\text { from Thailand } \\
(\mathrm{NC} 2)\end{array}$} & & $51,700,000$ & $43,733,333.33$ & $8,103,291.51$ & $4,678,437.53$ \\
\hline & 1 & $44,000,000$ & & & \\
\hline & & $\frac{35,500,000}{41,100000}$ & 4470000000 & 446766158 & 257940561 \\
\hline & 2 & $43,300,000$ & & & \\
\hline & & $49,700,000$ & & & \\
\hline & & $46,900,000$ & $49,000,000.00$ & $2,206,807.64$ & $1,274,100.99$ \\
\hline & 3 & $48,800,000$ & & & \\
\hline & & $51,300,000$ & & & \\
\hline \multirow{8}{*}{$\begin{array}{l}\text { N. ceranae from } \\
\text { A. mellifera } \\
\text { from France } \\
(\mathrm{NF})\end{array}$} & & $26,000,000$ & $25,066,666.67$ & $901,849.950$ & $520,683.311$ \\
\hline & 1 & $24,200,000$ & & & \\
\hline & & $25,000,000$ & & & \\
\hline & & $26,300,000$ & $25,766,666.67$ & $1,955,334.58$ & $1,128,912.94$ \\
\hline & 2 & $\begin{array}{l}27,400,000 \\
23,600,000\end{array}$ & & & \\
\hline & & $26,700,000$ & $25,166,666.67$ & $2,318,045.15$ & $1,338,323.99$ \\
\hline & 3 & $26,300,000$ & & & \\
\hline & & $22,500,000$ & & & \\
\hline \multirow[t]{9}{*}{ N. apis (NA) } & & $26,000,000$ & $20,366,666.7$ & $4,880,915.21$ & $2,817,997.71$ \\
\hline & 1 & $17,700,000$ & & & \\
\hline & & $17,400,000$ & & & \\
\hline & & $16,100,000$ & $17,466,666.7$ & $1,266,227.99$ & $731,057.073$ \\
\hline & 2 & $17,700,000$ & & & \\
\hline & & $18,600,000$ & & & \\
\hline & & $13,400,000$ & $15,500,000.00$ & $1,835,755.97$ & $1,059,874.20$ \\
\hline & 3 & $16,800,000$ & & & \\
\hline & & $16,300,000$ & & & \\
\hline
\end{tabular}

